\title{
Ring box protein-1 is associated with a poor prognosis and tumor progression in esophageal cancer
}

\author{
TOMOHIRO KUNISHIGE ${ }^{1}$, KAZUHIRO MIGITA ${ }^{1}$, SOHEI MATSUMOTO ${ }^{1}$, \\ KOHEI WAKATSUKI ${ }^{1}$, HIROSHI NAKADE ${ }^{1}$, SHINTARO MIYAO $^{1}$, \\ HIROKI KUNIYASU ${ }^{2}$ and MASAYUKI SHO ${ }^{1}$ \\ Departments of ${ }^{1}$ Surgery and ${ }^{2}$ Molecular Pathology, Nara Medical University, Kashihara, Nara 634-8522, Japan
}

Received December 24, 2019; Accepted May 28, 2020

DOI: $10.3892 / 01.2020 .11840$

\begin{abstract}
Ring box protein-1 (RBX1) is an essential component of the S-phase kinase-associated protein, Cullin and F-box containing ubiquitin ligases. Overexpression of RBX1 has been reported in several cancer types; however, little is known regarding the prognostic value and role of RBX1 in esophageal cancer. The present study examined 120 patients with esophageal cancer (EC) who underwent curative esophagectomy and 61 patients with EC who underwent neoadjuvant combination chemotherapy with docetaxel, cisplatin and 5-fluorouracil (5-FU; DCF) using immunohistochemistry. All specimens were classified into two groups according to the percentage of RBX1-positive tumor cells. In addition, the impact of RBX1 expression on cancer cell proliferation was analyzed in vitro using a small interfering RNA silencing technique. RBX1 expression levels showed significant differences according to tumor size $(\mathrm{P}<0.001)$, tumor depth $(\mathrm{P}=0.002)$, lymph node metastasis $(\mathrm{P}=0.004)$, pathological stage $(\mathrm{P}=0.001)$, lymphatic invasion $(\mathrm{P}=0.001)$ and venous invasion $(\mathrm{P}=0.001)$. The overall survival (OS) rate in the RBX1 high expression group was significantly lower compared with that in the low group $(\mathrm{P}=0.004)$. Multivariate analysis demonstrated that RBX1 status was an independent prognostic factor. $R B X I$ gene silencing inhibited the proliferation of human EC cells and enhanced the antitumor effect of 5-FU. Among patients who underwent neoadjuvant DCF therapy, the RBX1 high expression group had a significantly lower OS rate compared with that of the RBX1-low group ( $\mathrm{P}<0.001)$. In conclusion, RBX1 has notable prognostic value, and RBX1 may serve an important function in the tumor progression of EC.
\end{abstract}

\section{Introduction}

Esophageal cancer (EC) ranks seventh in terms of incidence and sixth in mortality rate worldwide for 36 cancers in 185

Correspondence to: Dr Tomohiro Kunishige, Department of Surgery, Nara Medical University, 840 Shijo-cho, Kashihara, Nara 634-8522, Japan

E-mail: tkuni0118@naramed-u.ac.jp

Key words: ring box protein-1, prognostic factor, proliferation, chemotherapy, esophageal cancer countries (1). A randomized controlled trial demonstrated that preoperative chemotherapy had a significantly higher survival rate compared with postoperative adjuvant chemotherapy in advanced EC (2,3). Based on the results of this previous trial, preoperative chemotherapy followed by esophagectomy has become a standard treatment strategy for advanced EC in Japan. However, the prognosis of patients with EC remains poor, with a 5-year OS rate of $43-61 \%$ (3-5). It is therefore necessary to develop novel strategies for the treatment of EC and to identify predictive factors for the outcomes of patients in order to improve treatment planning.

S-phase kinase-associated protein (Skp), Cullin and F-box containing (SCF) ubiquitin ligases contain Ring box protein-1 (RBX1), and are the largest E3 ubiquitin ligases. These ligases serve a crucial role in controlling protein turnover, and regulating various signaling pathways and cellular processes, such as cell cycle regulatory proteins, transcription factors and signal transducers, as part of the ubiquitin-proteasome systems (6-9). Thus, dysfunction of SCF ubiquitin ligases due to the aberrant expression of the complex components can cause various diseases, including cancer (10-12). RBX1 is an essential component of the SCF ubiquitin ligase complex, interacting with different members of the Cullin family of proteins and forming catalytic cores $(7,13)$. RBX1 is expressed in multiple human tissues, in particular the placenta, muscle, heart and liver (14). It has been reported that RBX1 is overexpressed in several cancer types, such as lung, breast, liver and gastric cancers, and RBX1 expression levels are significantly associated with the survival rate of patients with cancer $(15,16)$. Furthermore, RBX1 serves an important role in inducing cancer cell proliferation, including esophageal and gastric cancer $(15,17)$. Previously, dysfunction of E3 ubiquitin ligases has been reported to be associated with resistance to chemotherapy and radiotherapy, such as acute myelogenous leukemia and lung cancer (18-20). However, little is known regarding the prognostic value of $\mathrm{RBX} 1$ and the association between RBX1 expression and its effect on chemotherapy in $\mathrm{EC}$.

The present study investigated the clinical significance and prognostic impact of RBX1 expression in EC. Furthermore, the role of $\mathrm{RBX} 1$ in the progression of $\mathrm{EC}$ was evaluated in vitro using a small interfering (si)RNA silencing technique. 


\section{Materials and methods}

Patients. In total, 120 patients (97 men and 23 women) with squamous cell carcinoma who underwent R0 esophagectomy in the Department of Surgery, Nara Medical University Hospital between January 1995 and December 2011 were included in the present study. The median age of the patients was 63 years (age range, $42-80$ years). These patients had not undergone chemotherapy or radiotherapy before surgery. Cancerous and non-cancerous tissue specimens were obtained from resected specimens of 13 patients and were stored at $-80^{\circ} \mathrm{C}$. Non-cancerous tissues were obtained from adjacent normal tissues ( $>2 \mathrm{~cm}$ away from cancerous tissues). The rest of each specimen was fixed in $10 \%$ phosphate-buffered formalin at room temperature for $24-48 \mathrm{~h}$ and embedded in paraffin for immunohistochemical analysis. Tumors were classified according to the Tumor-Node-Metastasis (TNM) staging system (21). The patients were followed up until death or November 2018 in an outpatient clinic. The median follow-up period for all patients was 33.4 months. Furthermore, 61 patients with EC who underwent neoadjuvant combination chemotherapy with docetaxel, cisplatin and 5-fluorouracil (5-FU; DCF) and subsequent esophagectomy in the Department of Surgery, Nara Medical University Hospital, between January 2008 and December 2017 were also included. Among these patients, 60 had squamous cell carcinoma, and one had adenocarcinoma. These patients were followed up until death or November 2018 in an outpatient clinic. The median follow-up period for these patients was 44.2 months. The pathological response to chemotherapy was evaluated according to the Japanese Classification of Esophageal Cancer as follows: Grade 0 , no recognizable cytological or histological therapeutic effect; grade 1a, viable cancer cells accounting for $2 / 3$ or more of the tumor tissue; grade $1 b$, viable cancer cells accounting for $1 / 3$ or more, but $<2 / 3$, of the tumor tissue; grade 2 , viable cancer cells accounting for $<1 / 3$ of the tumor tissue, while other cancer cells are severely degenerated or necrotic; and grade 3, no viable cancer cells are evident (22). Written informed consent was provided by all patients prior to surgery, and the present study was approved by the Local Ethics Committee on Clinical Investigation of Nara Medical University (Kashihara, Japan; approval no. 1980).

Immunohistochemistry. Formalin-fixed, paraffin-embedded tissues were cut into $5 \mu \mathrm{m}$-thick-sections. Sections were stained using a DAKO EnVision system (Dako; Agilent Technologies, Inc.), as described previously $(16,23,24)$. Mouse monoclonal anti-RBX1 antibody (cat. no. ab133565, 1:250 dilution; Abcam) was used as the primary antibody. To evaluate RBX1 expression levels, at least 1,000 tumor cells from the invasive front of tumors were scored at $\times 400$ magnification, and the percentage of positively stained tumor cells was calculated. A cut-off value for percentage of RBX1-positive tumor cells was set at median value $(76.2 \%)$.

Cell lines. Human EC cell lines (TE-1 and TE-6) were obtained from the RIKEN BioResource Center and cultured in RPMI-1640 (Wako Pure Chemical Industries, Ltd.) supplemented with $10 \%$ heat-inactivated fetal bovine serum. All cells were maintained in an atmosphere containing $5 \% \mathrm{CO}_{2}$ at $37^{\circ} \mathrm{C}$.
Extraction of total $m R N A$ and reverse transcriptase-quantitative (RT-q)PCR. Total RNA was extracted from EC tissues, non-cancer tissues and human EC cell lines using RNAspin Mini (GE Healthcare), and the first-strand cDNA was synthesized from $1 \mu \mathrm{g}$ RNA using the high-capacity cDNA reverse transcription kit (cat. no. 4368813; Applied Biosystems; Thermo Fisher Scientific, Inc.) as previously described $(16,23,24)$. For RT-qPCR, cDNA was amplified using TaqMan Fast Universal PCR Master Mix (2X; Applied Biosystems; Thermo Fisher Scientific, Inc.) with gene-specific primers and a lysate probe on a StepOnePlus Real-Time PCR System (Applied Biosystems; Thermo Fisher Scientific, Inc.), according to the manufacturer's instructions. The thermal cycling conditions were $95^{\circ} \mathrm{C}$ for $20 \mathrm{sec}$, followed by 40 cycles of $95^{\circ} \mathrm{C}$ for $1 \mathrm{sec}$ and $60^{\circ} \mathrm{C}$ for 20 sec. qPCR experiments for each gene were carried out on 3 separate occasions. All primer/probe sets were purchased from Applied Biosystems; Thermo Fisher Scientific, Inc. (RBX1; Hs00360274m1, $\beta_{2}$-microglobulin; Hs00984230_m1). Expression levels of $\beta_{2}$-microglobulin, a housekeeping gene, were measured as an internal reference with a standard curve to determine the integrity of template RNA for all the specimens. The ratio of the RBX1 mRNA levels were calculated as follows: (Absolute copy number of RBX1)/(absolute copy number of $\beta_{2}$-microglobulin) (25).

Small interfering RNA (siRNA) transfection of RBXI. In transfection analyses, TE- 1 and TE- 6 cells were seeded in 6-well plates and transfected with either control RNA (scrambled siRNA; Qiagen, Inc.) or siRNA (20 nmol) against RBX1. Transfection was performed using the Lipofectamine ${ }^{\circledR}$ system (Invitrogen; Thermo Fisher Scientific), as described previously $(16,23,24)$. The human RBX1 siRNA duplexes, generated with 3'-dTdT overhangs and prepared by Qiagen, Inc, were used to target the following DNA sequence: 5'-CTG CTGTTACCTAATTACAAA-3'.

Cell viability assay. Cell viability was determined using a Cell-Titer Blue ${ }^{\circledR}$ Cell Viability Assay kit (Promega Corporation), as described previously $(16,23,24)$. Briefly, aliquots of $3.0 \times 10^{3}$ TE- 1 cells and $4.0 \times 10^{3}$ TE- 6 cells per well were cultured in 96-well plates at $37^{\circ} \mathrm{C}$. Cells were transfected with control RNA or RBX1 siRNA after $24 \mathrm{~h}$. Cell-Titer Blue reagent was added to each well $72 \mathrm{~h}$ after transfection. Fluorescence was measured at a wavelength of $590 \mathrm{~nm}$ upon excitation at $560 \mathrm{~nm}$ using a SoftMax ${ }^{\circledR}$ Pro 5 device (Molecular Devices, LLC). Each experiment was performed at least three times.

Cell cycle analysis. Cell cycle analysis was performed using a Cycletest ${ }^{\mathrm{TM}}$ Plus DNA Reagent kit (BD Biosciences), as described previously $(16,23,24)$. Cells were stained with propidium iodide at $4^{\circ} \mathrm{C}$ for 15 min using the Cycletest ${ }^{\mathrm{TM}}$ Plus DNA Reagent kit (cat. no. 340242; BD Biosciences). The cellular DNA content of at least $2 \times 10^{4}$ cells was analyzed using a FACSCalibur instrument (BD Biosciences), and the percentages of cells in the different phases of the cycle were determined using the CellQuest software program (version 6.0; BD Biosciences).

5-FU treatment. For the cytotoxicity assay, aliquots of $3.0 \times 10^{3}$ TE- 1 cells and $4.0 \times 10^{3}$ TE- 6 cells per well were seeded in 
96-well plates. After $24 \mathrm{~h}$, cells were treated with 5-FU (10 $\mu \mathrm{M}$; Wako Pure Chemical Industries, Ltd.), RBX1 siRNA (20 nmol; Qiagen, Inc.), or a combination of 5-FU and RBX1 siRNA at $37^{\circ} \mathrm{C}$. Control cells received no treatment. After $72 \mathrm{~h}$, cell viability was determined using a Cell-Titer Blue Cell Viability Assay kit. Each experiment was performed at least three times.

Western blotting. Human EC cell lines (TE-1 and TE-6) transfected with control RNA or RBX1 siRNA were harvested, and whole cell proteins were exracted using PRO-PREP ${ }^{\mathrm{TM}}$ Protein Extraction Solution for Cell/Tissue (Intron Biotechnology, Inc.), and densities were measured using a DeNovix DS-11 spectrophotometer (DeNovix Inc., https://www.denovix.com). Proteins $(15 \mu \mathrm{g})$ were loaded per lane onto a 10 or $12 \%$ gels, resolved using SDS-PAGE and then transferred onto polyvinylidene difluoride membranes (EMD Millipore), which were then blocked using 5\% skimmed milk at room temperature for $1 \mathrm{~h}$. Mouse monoclonal anti-RBX1 antibodies (cat. no. ab133565; 1:5,000 dilution; Abcam), rabbit monoclonal anti-p21 antibodies (cat. no. sc-397; 1:100 dilution; Santa Cruz Biotechnology, Inc.) and mouse monoclonal anti-actin antibodies (cat. no. 3700, 1:1,000 dilution; Cell Signaling Technology, Inc.) were used. The membranes were incubated with these primary antibodies overnight at $4^{\circ} \mathrm{C}$, and then were incubated with horseradish peroxidase-conjugated anti-mouse or anti-rabbit immunoglobulin $\mathrm{G}$ secondary antibodies (anti-mouse IgG-HRP; cat. no. sc-2005; 1:5,000 dilution and anti-rabbit IgG-HRP; cat. no. sc-2004; 1:5,000 dilution; both purchased from Santa Cruz Biotechnology, Inc.) at room temperature for $1 \mathrm{~h}$. Peroxidase activity was detected on X-ray films using an enhanced chemiluminescence detection system (Western Lightning Plus-ECL; PerkinElmer, Inc.).

Statistical analysis. Continuous variables are expressed as the mean \pm standard deviation, and the means were compared using an unpaired t-test or a one-way ANOVA with Tukey's post hoc test. Categorical variables are presented as numbers and percentages, and groups were compared using the $\chi^{2}$ test or Fisher's exact test. Overall survival (OS) was defined as the time from the operation until death. Relapse-free survival (RFS) was defined as the time from the operation until EC recurrence. Survival curves were constructed using the Kaplan-Meier method, and differences between the curves were analyzed using a log-rank test. Univariate and multivariate hazard ratios (HRs) were calculated using the Cox proportional hazards model. All significant variables identified in the univariate analysis were entered into a multivariate analysis. $\mathrm{P}<0.05$ was considered to indicate a statistical significance and confidence intervals (CIs) were calculated at the $95 \%$ level. All statistical analyses were performed using SPSS version 19.0 (IBM Corp.).

\section{Results}

$R B X 1$ expression in $E C$. The relative expression levels of $\mathrm{RBX} 1$ between EC tissues and non-cancer tissues were compared using frozen tissues. qPCR indicated that 11 of 13 (84.6\%) EC tissues expressed higher levels of RBX1 mRNA compared with those of non-cancer tissues. In addition, EC tissues expressed signifi-
Table I. Association between the clinicopathological factors and RBX1 expression levels of patients with esophageal cancer.

\begin{tabular}{|c|c|c|c|}
\hline Variables & Value $(\%)$ & $\begin{array}{l}\text { Percentage of } \\
\text { RBX1-positive } \\
\text { tumor cells }(\%)\end{array}$ & $\mathrm{P}$-value ${ }^{\mathrm{a}}$ \\
\hline Age, years & & & 0.067 \\
\hline$<63$ & $58(48.3)$ & $60.8 \pm 23.8$ & \\
\hline$\geq 63$ & $62(51.7)$ & $69.1 \pm 25.2$ & \\
\hline Sex & & & 0.926 \\
\hline Male & $97(80.8)$ & $65.0 \pm 25.1$ & \\
\hline Female & $23(19.2)$ & $65.5 \pm 24.1$ & \\
\hline Tumor size, $\mathrm{mm}$ & & & $<0.001$ \\
\hline$<50$ & $79(65.8)$ & $60.0 \pm 26.2$ & \\
\hline$\geq 50$ & $41(34.2)$ & $75.0 \pm 18.6$ & \\
\hline Tumor depth & & & 0.002 \\
\hline $\mathrm{T} 1 / \mathrm{T} 2$ & $61(50.8)$ & $58.5 \pm 27.3$ & \\
\hline $\mathrm{T} 3 / \mathrm{T} 4$ & $59(49.2)$ & $72.0 \pm 20.0$ & \\
\hline Lymph node metastasis & & & 0.004 \\
\hline Negative & $48(40.0)$ & $56.8 \pm 27.3$ & \\
\hline Positive & $72(60.0)$ & $70.6 \pm 21.4$ & \\
\hline Distant metastasis & & & 0.433 \\
\hline No & $111(92.5)$ & $64.6 \pm 25.4$ & \\
\hline Yes & $9(7.5)$ & $71.4 \pm 14.2$ & \\
\hline Pathological stage & & & 0.001 \\
\hline $\mathrm{I} / \mathrm{II}$ & $57(47.5)$ & $57.1 \pm 27.5$ & \\
\hline III/IV & $63(52.5)$ & $72.4 \pm 19.6$ & \\
\hline Lymphatic invasion & & & 0.001 \\
\hline Negative & $34(28.3)$ & $51.7 \pm 27.6$ & \\
\hline Positive & $86(71.7)$ & $70.4 \pm 21.6$ & \\
\hline Venous invasion & & & 0.001 \\
\hline Negative & $74(61.7)$ & $59.6 \pm 26.3$ & \\
\hline Positive & $46(38.3)$ & $74.0 \pm 19.3$ & \\
\hline
\end{tabular}

Values are expressed as the mean \pm standard deviation. ${ }^{\text {aValue }}$ obtained using a Student's t-test. RBX1, ring box protein-1.

cantly higher levels of RBX1 mRNA compared with non-cancer tissues $(\mathrm{P}<0.001$; Fig. 1A). The protein expression of RBX1 was then investigated (Fig. 1B). In all EC tissue specimens, positive staining for RBX1 was detected in the nuclei of cancer cells. In some cancer cells, the cytoplasm was also positively stained. Normal squamous epithelial cells showed a lack of staining, and positive staining was observed in some mononuclear cells. Overall, the median percentage of RBX1-positive tumor cells was $76.2 \%$ (range, $2.5-93.5 \%$; data not shown).

Clinicopathological significance of RBX1 expression in EC. The associations between clinicopathological factors and RBX1 expression are shown in Table I. There were significant differences in the percentage of RBX1-positive tumor cells in terms of the tumor size ( $<50$ vs. $\geq 50 \mathrm{~mm}, \mathrm{P}<0.001)$, tumor depth (T1/T2 vs. T3/T4, P=0.002) and pathological stage 

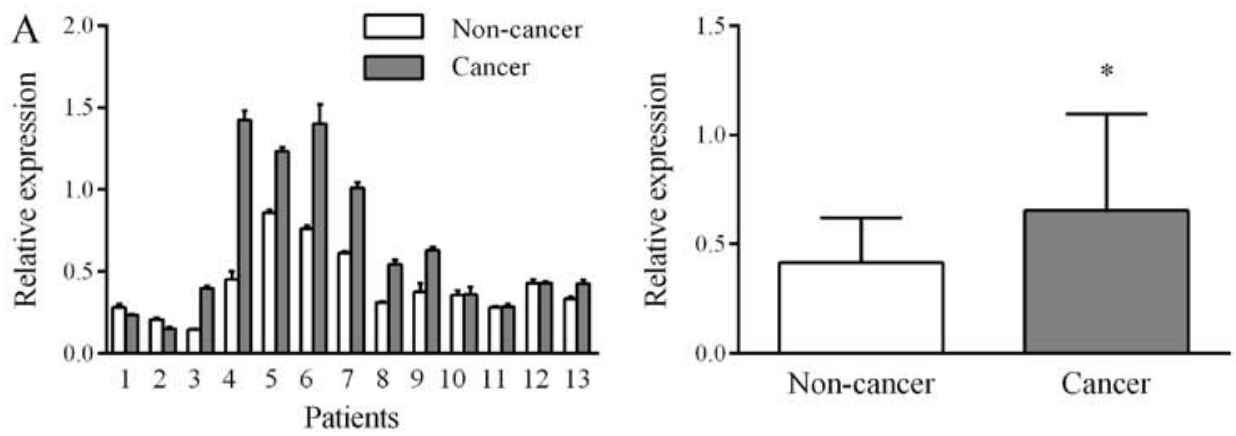

B
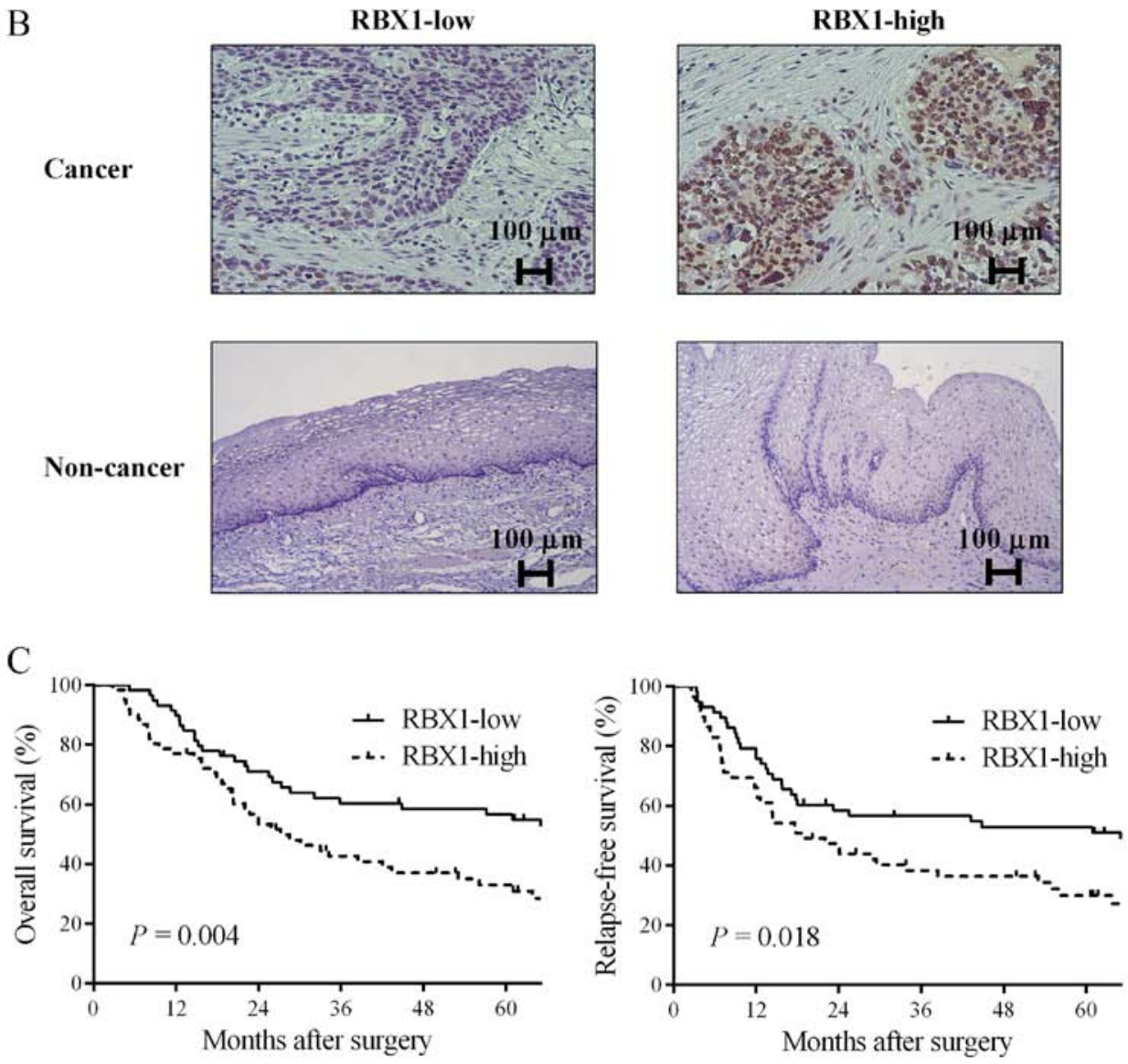

Figure 1. Clinical significance of RBX1 expression in EC. (A) Expression of RBX1 in cancer tissue was higher compared with non-cancer tissue in 84.6\% of patients with EC. RBX1 expression of cancer tissues specimens $(n=13)$ was significantly higher compared with that of non-cancer tissues. (B) Representative cases with low and high RBX1 expression levels. Magnification, $x 200$. (C) Postoperative overall $(\mathrm{P}=0.004)$ and relapse-free survival $(\mathrm{P}=0.018)$ in patients with RBX1 high expression tumors were significantly poorer compared with patients with RBX1 low expression tumors. "P $<0.05$ vs. non-cancer tissues. EC, esophageal cancer; RBX1, ring box protein-1.

(stage I/II vs. III/IV, $\mathrm{P}=0.001$ ). Tumors with lymph node metastasis $(\mathrm{P}=0.004)$, lymphatic invasion $(\mathrm{P}=0.001)$ and venous invasion $(\mathrm{P}=0.001)$ had significantly higher positive numbers of RBX1 cells compared with those without such factors.

Association of RBX1 expression with postoperative prognosis. To investigate the prognostic significance of RBX1 expression in EC, all specimens were classified into two groups according to the percentage of RBX1-positive tumor cells. In total, 61 (50.8\%) patients with an RBX1-positive rate of $\geq 76.2 \%$ were classified into the RBX1 high expression group, and 59 (49.2\%) patients with an RBX1-positive rate of $<76.2 \%$ were classified into the RBX1 low expression group (Fig. 1B). The 5-year OS rate of the RBX1 high expression group was significantly lower compared with that of the low expression group (33.0 vs. $56.7 \%$, respectively, $\mathrm{P}=0.004$; Fig. 1C). There was also a significant difference in the 5-year RFS rate between the RBX1 high expression and low expression groups (30.0 vs. $52.9 \%$, respectively, $\mathrm{P}=0.018$; Fig. $1 \mathrm{C}$ ).

Influence of RBXI expression on postoperative recurrence. At the time of the analysis, 62 patients $(51.7 \%)$ had postoperative recurrence. Some patients relapsed after 5 years and died due to other reasons. Overall, there was no significant difference in the recurrence rate between the high and low RBX1 expression groups $(\mathrm{P}=0.101$; Table II). With regard to the site of recurrence, the RBX1 high expression group had a significantly higher rate of lymph node recurrence compared with the low 
A

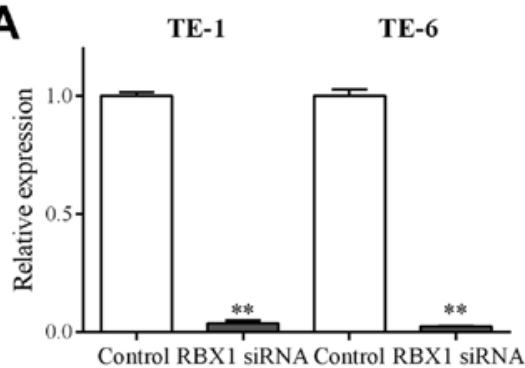

C

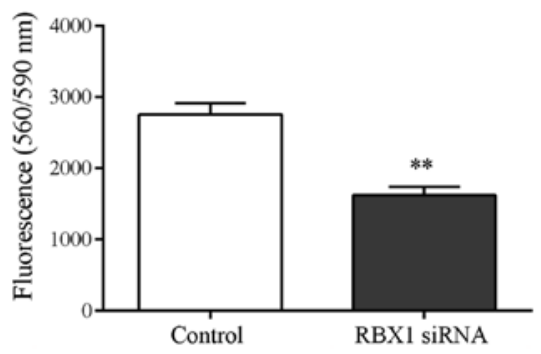

D TE-1
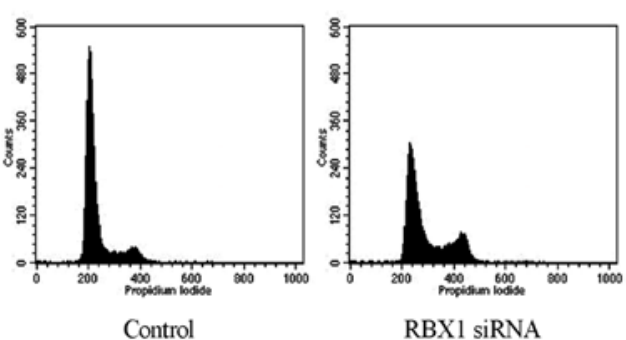

TE-1

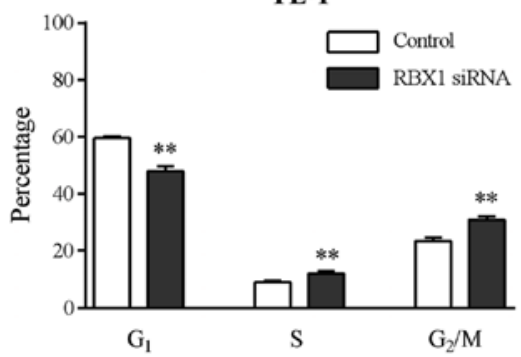

B

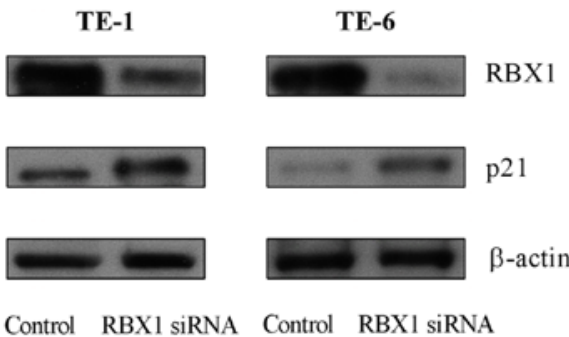

TE-6

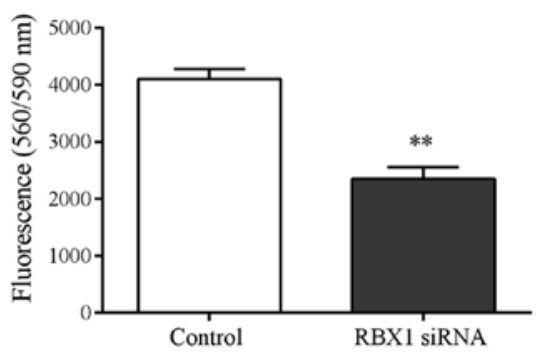

TE-6

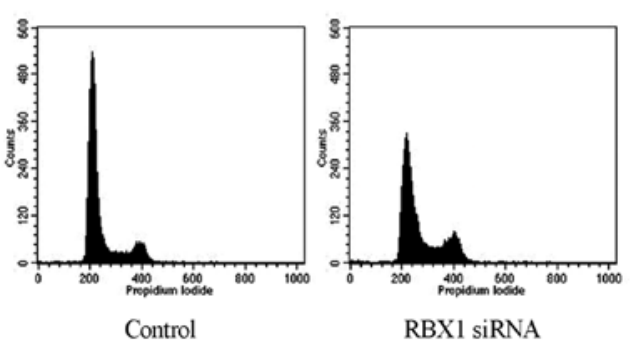

TE-6

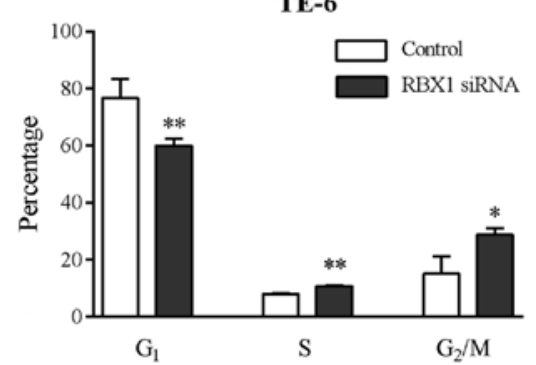

Figure 2. Downregulation of RBX1 expression using siRNA inhibits cell proliferation and induces cell cycle arrest in esophageal cancer. (A) TE-1 and TE-6 cells were transfected with control RNA or RBX1 siRNA. RBX1 expression was evaluated using reverse-transcription quantitative PCR. After transfection with RBX1 siRNA, expression of RBX1 mRNA was significantly reduced for up to $72 \mathrm{~h}$ in both cell lines. (B) Protein levels of RBX1, p21 and $\beta$-actin were analyzed using western blotting. (C) Cell proliferation was significantly inhibited in cells treated with RBX1 siRNA, as determined using a CellTiter-Blue ${ }^{\circledR}$ Cell Viability assay after $72 \mathrm{~h}$ of incubation ( $\mathrm{n}=10$ from each group). (D) TE1 and TE6 cells were transfected with siRNA for $72 \mathrm{~h}$, and then subjected to propidium iodide staining. The cell cycle profile was analyzed using flow cytometry. ${ }^{*} \mathrm{P}<0.05,{ }^{* *} \mathrm{P}<0.01$ vs. control. RBX1, ring box protein-1; si, small interfering.

expression group $(\mathrm{P}=0.007$; Table II). There were no significant differences in pleural and hematogenous recurrence rates between the high and low RBX1 expression groups.

Prognostic value of the RBX1 expression in EC. In the univariate analysis, the factors that were significantly associated with OS were sex $(\mathrm{P}=0.022)$, tumor depth $(\mathrm{P}<0.001)$, lymph node metastasis $(\mathrm{P}<0.001)$, distant metastasis $(\mathrm{P}=0.002)$, venous invasion $(\mathrm{P}=0.013)$ and $\mathrm{RBX} 1$ expression $(\mathrm{P}=0.004$; data not shown). Multivariate analysis demonstrated that $\mathrm{RBX} 1$ expression $(\mathrm{P}=0.012)$, as well as sex $(\mathrm{P}=0.006)$, tumor depth $(\mathrm{P}<0.001)$ and lymph node metastasis $(\mathrm{P}=0.001)$ were independent prognostic factors for OS (Table III).
Association between RBX1 expression and proliferation of EC cells. As previous studies have suggested the involvement of RBX1 in cancer cell proliferation (15-17), the present study investigated the effect of RBX1 downregulation on the proliferation of EC cells. RBX1 downregulation was induced in human EC cell lines (TE-1 and TE-6) by using an siRNA knockdown approach. mRNA and protein expression levels of RBX1 were significantly reduced by RBX1 siRNA compared with the control RNA in both cell lines $72 \mathrm{~h}$ post-transfection $(\mathrm{P}<0.001$; Fig. 2A and B). The CellTiter-Blue Cell Viability assay showed that RBX1 gene silencing significantly inhibited cell proliferation for up to $72 \mathrm{~h}$ in both cell lines $(\mathrm{P}<0.001$; Fig. 2C). 

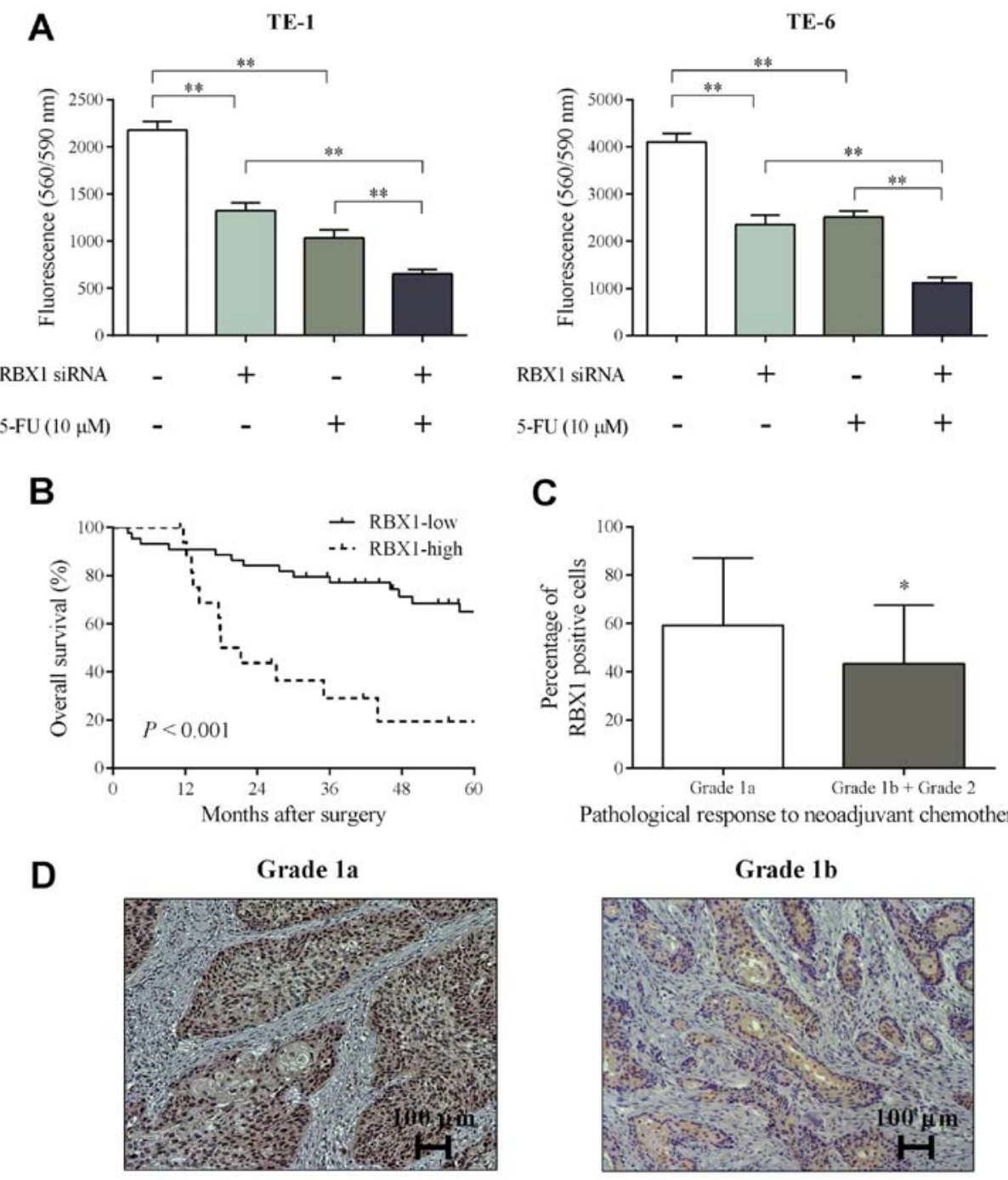

Pathological response to neoadjuvant chemotherapy

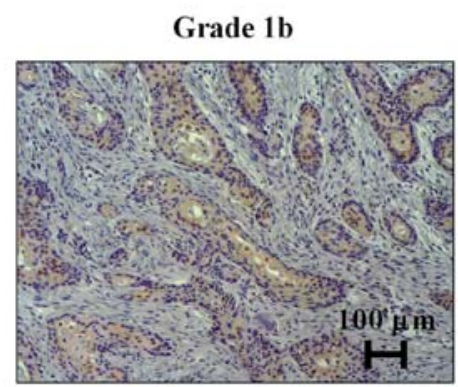

Figure 3. Associations between the expression levels of RBX1 and the antitumor effect of chemotherapy. (A) Cell proliferation was significantly inhibited by combined treatment with 5-FU and RBX1 siRNA in comparison to 5-FU or RBX1 siRNA alone, as determined using a CellTiter-Blue ${ }^{\circledR}$ Cell Viability assay after $72 \mathrm{~h}$ of incubation ( $\mathrm{n}=10$ from each group). (B) Among patients who received neoadjuvant combination chemotherapy with DCF, postoperative overall survival in patients with high RBX1 expression in tumors was significantly poorer compared with patients with low expression tumors. (C) Mean percentage of RBX1-positive tumor cells was significantly higher in tumors with a grade 1a compared with tumors with grade $1 \mathrm{~b}$ or 2 pathological responses. (D) Representative cases of RBX1 expression according to the pathological response to neoadjuvant DCF therapy. Magnification, x200. "P<0.05 vs. grade 1a; ${ }^{* *} \mathrm{P}<0.01$. RBX1, ring box protein-1; si, small interfering; DCF, docetaxel, cisplatin and 5-fluorouracil.

Table II. Impact of the RBX1 status on postoperative recurrence.

\section{RBX1 expression}

\begin{tabular}{lccc} 
Site & Low, $\mathrm{n}=59(\%)$ & High, $\mathrm{n}=61(\%)$ & P-value \\
\hline All recurrence & $26(44.1)$ & $36(59.0)$ & $0.101^{\mathrm{a}}$ \\
Pleura & $1(1.7)$ & $5(8.5)$ & $0.107^{\mathrm{b}}$ \\
Hematogenous & $19(32.8)$ & $15(25.4)$ & $0.382^{\mathrm{a}}$ \\
Lymph node & $12(20.7)$ & $26(44.1)$ & $0.007^{\mathrm{a}}$
\end{tabular}

A total of 25 patients had first recurrence at $>1$ site. ${ }^{a}$ Obtained using a $\chi^{2}$ test; ${ }^{b}$ Obtained using a Fisher's exact test. RBX1, ring box protein-1.

RBX1 gene silencing induces $G_{2} / M$ cell cycle arrest in EC cells. In order to determine the mechanisms underlying the inhibition of cell proliferation by RBX1 gene silencing, the cell cycle profile of cells treated with RBX1 siRNA was examined. As shown in Fig. 2D, $R B X 1$ gene-silenced cells significantly increased the number of cells in the $\mathrm{G}_{2} / \mathrm{M}$ phase in both cell lines (TE-1, control RNA vs. RBX1 siRNA, $23.5 \pm 0.4$ vs. $30.9 \pm 0.5 \%$, $\mathrm{P}<0.001$; TE-6, $15.0 \pm 2.7$ vs. $28.8 \pm 1.0 \%, \mathrm{P}=0.002)$. Western blotting showed that the protein levels of $\mathrm{p} 21$ were increased by $R B X 1$ gene silencing in both cell lines (Fig. 2B).

Efficacy of chemotherapy combination with RBXI gene silencing. The association between RBX1 expression levels and the antitumor effect of chemotherapeutic agents was investigated, as SCF E3 ligases are associated with chemotherapy resistance $(19,20)$. Treatment with 5-FU or RBX1 siRNA alone significantly inhibited the proliferation of EC cells compared with the untreated control in both cell lines $(\mathrm{P}<0.001$; Fig. $3 \mathrm{~A})$. In addition, the combination of 5-FU and RBX1 siRNA treatment significantly inhibited the proliferation of EC cells compared with 5-FU or RBX1 siRNA alone in both cell lines ( $\mathrm{P}<0.001$; Fig. 3A). 
Table III. Results of the multivariate analysis for overall survival.

\begin{tabular}{llr}
\hline Variables & Hazard ratio (95\% CI) & P-value \\
\hline Age, years & $0.968(0.934-1.003)$ & 0.071 \\
Sex, male vs. female & $2.771(1.339-5.737)$ & 0.006 \\
Tumor depth, T3/T4 vs. T1/T2 & $3.040(1.760-5.250)$ & $<0.001$ \\
Lymph node metastasis, positive vs. negative & $2.703(1.520-4.808)$ & 0.001 \\
Distant metastasis, yes vs. no & $2.030(0.968-4.257)$ & 0.061 \\
Venous invasion, positive vs. negative & $0.950(0.551-1.638)$ & 0.854 \\
RBX1, high vs. low & $2.023(1.165-3.513)$ & 0.012
\end{tabular}

Simultaneously adjusted for age, sex, tumor depth, lymph node metastasis, distant metastasis, venous invasion and RBX1. CI, confidence interval; RBX1, ring box potein-1.

Association between postoperative survival and RBXI expression in patients with EC who underwent neoadjuvant chemotherapy. Lastly, the prognostic value of RBX1 in patients who underwent neoadjuvant DCF therapy followed by curative esophagectomy was examined. The 5-year OS rate of patients with tumors with high RBX1 expression was significantly lower compared with that of patients with low RBX1 expression (19.4 vs. $65.0 \%, \mathrm{P}<0.001$, Fig. 3B). Furthermore, the mean percentage of RBX1-positive tumor cells was significantly higher in grade la tumors compared with grade $1 \mathrm{~b}$ or 2 tumors $(\mathrm{P}<0.05$; Fig. 3C and D).

\section{Discussion}

The present study investigated the expression of RBX1, a core subunit of SCF ubiquitin ligases (6-9), and demonstrated its clinical importance in EC. Previous studies have reported that overexpression of components of the SCF complex, such as Skp2, Cullin1 and 4, were significantly associated with a worse prognosis in several cancer types, such as gastric cancer and breast cancer $(12,23,26-28)$. Similarly, some studies evaluated the prognostic value of RBX1 in cancer, and identified that higher expression of RBX1 is an independent factor for poor prognosis in gastric cancer and non-muscle-invasive bladder transitional cell carcinoma $(16,29)$. Recently, Zhang et al (15) have also shown that the overexpression of RBX1 is significantly associated with a lower OS rate in EC. In the present study, the mRNA levels of RBX1 were higher in EC tissues compared with those in non-cancer tissues; however, pairs of cancerous and non-cancerous tissues were only available for 13 patients. Immunohistochemical analysis also showed that RBX1 was overexpressed in EC tissues compared with adjacent normal tissues, and the higher expression of RBX1 was significantly associated with worse postoperative OS. Importantly, RBX1 expression levels were identified as an independent predictor of OS. These results suggested that RBX1 serves as a novel prognostic biomarker, and highlights the clinical importance of RBX1 in EC.

A limited number of studies have investigated the clinical significance of RBX1 in cancer. Some studies have shown significant associations between the expression levels of RBX1 and various tumor-associated factors, such as tumor depth and tumor size. One study reported that the expression levels of RBX1 were positively associated with tumor grade and stage in non-muscle-invasive bladder transitional cell carcinoma (29). Another study showed that the expression levels of RBX1 were significantly associated with tumor size and pathological differentiation grade in hepatocellular carcinoma (30). Our previous study reported that the expression levels of RBX1 were significantly associated with tumor depth, distant metastasis and venous invasion in gastric cancer (16). The present study revealed that a higher expression of RBX1 was significantly associated with advanced tumor features, including larger tumor size, deeper depth of invasion, lymph node metastasis, advanced pathological stage, lymphatic invasion and venous invasion. These results indicate that RBX1 might be involved in promoting the progression and metastasis of EC.

The present study also evaluated the impact of the RBX1 expression on postoperative recurrence in EC. Some studies have investigated the association between the expression of RBX1 and cancer recurrence. Wang et al (29) demonstrated that the expression levels of RBX1 were independently associated with local recurrence of non-muscle-invasive bladder transitional cell carcinoma. Our previous study showed that patients with tumors with higher expression of RBX1 had a significantly higher rate of hematogenous recurrence in gastric cancer (16). In the present study, the 5-year RFS rate in the RBX1 high expression group was significantly lower compared with that in the low expression group. In addition, lymph node recurrence was significantly more common in patients with high RBX1 expression compared with patients with low expression. This higher rate of lymph node recurrence may be partly due to the higher frequency of lymph node metastasis and lymphatic invasion in tumors with high RBX1 expression. These findings suggest that RBX1 status can be a useful predictor of recurrence in patients with EC.

It has been reported that RBX1 is essential for cancer cell proliferation and survival, such as colon cancer and breast cancer cells $(31,32)$. In the present study, the effect of the RBX1 expression on the proliferation of EC cells was examined. Zhang et al (15) showed that knockdown of RBX1 expression significantly inhibited the proliferation of EC cells. Similarly, the present study demonstrated that $R B X 1$ gene silencing significantly inhibited the proliferation of EC cells. The mechanism by which $R B X 1$ gene silencing inhibits the proliferation 
of EC cells was then further investigated. It is well known that SCF ligases ubiquitinate and degrade various cell cycle regulators, such as p21 and p27 (12). Previous studies have shown that $R B X 1$ gene silencing leads to $\mathrm{G}_{2} / \mathrm{M}$ cell cycle arrest and the accumulation of p21 and p27 proteins $(15,17,31)$. Consistent with these results, the present study showed that $R B X I$ gene silencing induced $\mathrm{G}_{2} / \mathrm{M}$ cell cycle arrest and increased the protein levels of p21. p21 is known to inhibit the activity of cyclin-dependent kinase and cyclin complexes and block cell cycle progression (33). The present study did not investigate these protein levels, such as p27 and cyclin B; however, these results suggested that RBX1 might stimulate the cell cycle, partly through the regulation of the p21 level in EC

In clinical practice, the prediction of responses to anticancer treatment is important for improving oncological outcomes. Some studies have evaluated the association between the clinical outcomes and expression levels of E3 ubiquitin ligases, and have shown that overexpression of E3 ubiquitin ligases, such as RNF126 and Nrdp1, is associated with a poor prognosis, and higher rates of metastasis and/or recurrence in patients with breast cancer who underwent adjuvant chemotherapy $(34,35)$. Tian et al (36) reported that high expression of Skp2 was associated with lower rates of disease-specific survival, local recurrence-free survival and metastasis-free survival in patients with rectal cancer who received preoperative chemoradiotherapy. However, the clinical significance of RBX1 in patients with cancer who have undergone chemotherapy and/or radiotherapy remains unclear. Furthermore, the association between RBX1 expression and pathological response in patients who have undergone neoadjuvant chemotherapy is also unclear. In the present study, the OS rate of patients with high RBX1 expression tumors was significantly lower compared with that of patients with low expression, among patients who received neoadjuvant DCF therapy followed by curative esophagectomy. Furthermore, the positive rate of RBX1 in tumors with a grade la pathological response was significantly higher compared with that in tumors with grade $1 \mathrm{~b}$ or 2 pathological response. These data suggested that RBX1 may be a useful predictor of the response to chemotherapy.

It was hypothesized that chemotherapy may influence the expression levels of RBX1. Thus, the patients were divided into the without preoperative chemotherapy and with neoadjuvant DCF therapy group in the present study, and the expression levels of RBX1 in each group were evaluated. The present study could not evaluate RBX1 expression in specimens before chemotherapy; thus, further investigations on RBX1 expression before and after chemotherapy are required.

Chemotherapy resistance can lead to tumor recurrence and cause a worse prognosis in patients with cancer $(3,37)$. Thus, further enhancement of the antitumor effects of chemotherapy is required to prolong the survival of patients with EC. Dysfunction of E3 ubiquitin ligases has been suggested to contribute to chemoresistance $(15,19,20)$. Previous studies have reported that the inactivation of SCF ubiquitin ligases by the NEDD8-activating enzyme inhibitor, MLN4924, augments the efficacy of cytarabine in acute myelogenous leukemia and cisplatin in ovarian cancer $(19,20)$. Zhang et al (15) showed that knockdown of RBX1 expression enhanced the cytotoxicity of cisplatin in EC. In the present study, the combination of 5-FU and RBXI gene silencing resulted in a significant reduction in the proliferation of EC cells compared with 5-FU or $R B X 1$ gene silencing alone. These data suggested that $R B X 1$ silencing could serve as an attractive chemosensitizing method. Taken together, RBX1 could be an attractive therapeutic target for EC. However, further investigations are required to clarify the mechanisms underlying the precise function of RBX1 in the progression of EC.

In conclusion, the present study demonstrated that RBX1 is associated with features of advanced EC tumors, and that RBX1 has prognostic value in EC. Furthermore, RBX1 may have an important role in regulating the proliferation of EC cells and contribute to chemoresistance. The present data may provide a rationale for developing a novel therapeutic strategy targeting RBX1 in EC.

\section{Acknowledgements}

Not applicable.

\section{Funding}

The current study was supported by the Japan Society for the Promotion of Science KAKENHI (grant no. 19K18128).

\section{Availability of data and materials}

The datasets used and/or analyzed during the present study are available from the corresponding author upon reasonable request.

\section{Authors' contributions}

TK, KM, HK and MS conceived and designed the present study. TK, KM, SMa, KW, HN and SMi performed the experiments and acquired the data. TK and KM analyzed the data, whilst TK, KM, HK and MS interpreted the data. All authors read and approved the final manuscript.

\section{Ethics approval and consent to participate}

This study was approved by The Ethics Committee of Nara Medical University (Kashihara, Japan; approval no. 1980). All patients provided informed written consent.

\section{Patient consent for publication}

Not applicable.

\section{Competing interests}

The authors declare that they have no competing interests.

\section{References}

1. Bray F, Ferlay J, Soerjomataram I, Siegel RL, Torre LA and Jemal A: Global cancer statistics 2018: GLOBOCAN estimates of incidence and mortality worldwide for 36 cancers in 185 countries. CA Cancer J Clin 68: 394-424, 2018.

2. Ando N, Iizuka T, Ide H, Ishida K, Shinoda M, Nishimaki T, Takiyama W, Watanabe $\mathrm{H}$, Isono K, Aoyama N, et al; Japan Clinical Oncology Group: Surgery plus chemotherapy compared with surgery alone for localized squamous cell carcinoma of the thoracic esophagus: A Japan Clinical Oncology Group Study--JCOG9204. J Clin Oncol 21: 4592-4596, 2003. 
3. Ando N, Kato H, Igaki H, Shinoda M, Ozawa S, Shimizu H, Nakamura T, Yabusaki H, Aoyama N, Kurita A, et al: A randomized trial comparing postoperative adjuvant chemotherapy with cisplatin and 5-fluorouracil versus preoperative chemotherapy for localized advanced squamous cell carcinoma of the thoracic esophagus (JCOG9907). Ann Surg Oncol 19: 68-74, 2012.

4. Allum WH, Stenning SP, Bancewicz J, Clark PI and Langley RE: Long-term results of a randomized trial of surgery with or without preoperative chemotherapy in esophageal cancer. J Clin Oncol 27: 5062-5067, 2009.

5. van Hagen P, Hulshof MC, van Lanschot JJ, Steyerberg EW, van Berge Henegouwen MI, Wijnhoven BP, Richel DJ Nieuwenhuijzen GA, Hospers GA, Bonenkamp JJ, et al; CROSS Group: Preoperative chemoradiotherapy for esophageal or junctional cancer. N Engl J Med 366: 2074-2084, 2012.

6. Hershko A and Ciechanover A: The ubiquitin system. Annu Rey Biochem 67: 425-479, 1998.

7. Ohta T, Michel JJ, Schottelius AJ and Xiong Y: ROC1, a homolog of APC11, represents a family of cullin partners with an associated ubiquitin ligase activity. Mol Cell 3: 535-541, 1999.

8. Kamura T, Conrad MN, Yan Q, Conaway RC and Conaway JW: The Rbx1 subunit of SCF and VHL E3 ubiquitin ligase activates Rub1 modification of cullins Cde53 and Cul2. Genes Dev 13 2928-2933, 1999.

9. Tan P, Fuchs SY, Chen A, Wu K, Gomez C, Ronai Z and Pan ZQ Recruitment of a ROC1-CUL1 ubiquitin ligase by Skp1 and HOS to catalyze the ubiquitination of I kappa B alpha. Mol Cell 3 : 527-533, 1999.

10. Nakayama KI and Nakayama K: Ubiquitin ligases: Cell-cycle control and cancer. Nat Rev Cancer 6: 369-381,2006.

11. Petroski MD and Deshaies RJ: Function and regulation of cullin-RING ubiquitin ligases. Nat Rev Mol Cell Biol 6: 9-20, 2005

12. Frescas D and Pagano M: Deregulated proteolysis by the F-box proteins SKP2 and beta-TrCP: Tipping the scales of cancer. Nat Rev Cancer 8: 438-449, 2008.

13. Ohta T, Michel JJ and Xiong Y: Association with cullin partners protects ROC proteins from proteasome-dependent degradation. Oncogene 18: 6758-6766, 1999.

14. Swaroop M, Gosink M and Sun Y: SAG/ROC2/Rbx2/Hrt2, a component of SCF E3 ubiquitin ligase: Genomic structure, a splicing variant, and two family pseudogenes. DNA Cell Biol 20: 425-434, 2001.

15. Zhang J, Li S, Shang Z, Lin S, Gao P, Zhang Y, Hou S, Mo S, Cao W, Dong Z, et al: Targeting the overexpressed ROC1 induces G2 cell cycle arrest and apoptosis in esophageal cancer cells. Oncotarget 8: 29125-29137, 2017.

16. Migita K, Takayama T, Matsumoto S, Wakatsuki K, Tanaka T, Ito M, Nishiwada $S$ and Nakajima Y: Prognostic impact of RING box protein-1 (RBX1) expression in gastric cancer. Gastric Cancer 17: 601-609, 2014.

17. Jia L, Soengas MS and Sun Y: ROC1/RBX1 E3 ubiquitin ligase silencing suppresses tumor cell growth via sequential induction of G2-M arrest, apoptosis, and senescence. Cancer Res 69: 4974-4982, 2009.

18. Wei D, Morgan MA and Sun Y: Radiosensitization of cancer cells by inactivation of cullin-RING E3 ubiquitin ligases. Transl Oncol 5: 305-312, 2012

19. Nawrocki ST, Kelly KR, Smith PG, Espitia CM, Possemato A, Beausoleil SA, Milhollen M, Blakemore S, Thomas M, Berger A, et al: Disrupting protein NEDDylation with MLN4924 is a novel strategy to target cisplatin resistance in ovarian cancer. Clin Cancer Res 19: 3577-3590, 2013.

20. Nawrocki ST, Kelly KR, Smith PG, Keaton M, Carraway H, Sekeres MA,MaciejewskiJPand Carew JS:The NEDD8-activating enzyme inhibitor MLN4924 disrupts nucleotide metabolism and augments the efficacy of cytarabine. Clin Cancer Res 21: 439-447, 2015 .

21. Sobin LH and Compton CC: TNM seventh edition: what's new, what's changed: Communication from the International Union Against Cancer and the American Joint Committee on Cancer. Cancer 116: 5336-5339, 2010.
22. Japan Esophageal Society: Japanese Classification of Esophagea Cancer, 11th Edition: Part I. Esophagus 14: 1-36, 2017.

23. Nakade H, Migita K, Matsumoto S, Wakatsuki K, Kunishige T, Miyao S and Sho M: Overexpression of Cullin4A correlates with a poor prognosis and tumor progression in esophageal squamous cell carcinoma. Int J Clin Oncol 25: 446-455, 2020.

24. Ito $M$, Migita $K$, Matsumoto S, Wakatsuki $K$, Tanaka $T$, Kunishige T, Nakade $H$, Nakatani $M$ and Nakajima $Y$ : Overexpression of E3 ubiquitin ligase tripartite motif 32 correlates with a poor prognosis in patients with gastric cancer. Oncol Lett 13: 3131-3138, 2017.

25. Livak KJ and Schmittgen TD: Analysis of relative gene expression data using real-time quantitative PCR and the 2(-Delta Delta C(T)) Method. Methods 25: 402-408, 2001.

26. Masuda TA, Inoue H, Sonoda H, Mine S, Yoshikawa Y, Nakayama K, Nakayama K and Mori M: Clinical and biological significance of S-phase kinase-associated protein 2 (Skp2) gene expression in gastric carcinoma: Modulation of malignant phenotype by Skp2 overexpression, possibly via p27 proteolysis. Cancer Res 62: 3819-3825, 2002

27. Bai J, Zhou Y, Chen G, Zeng J, Ding J, Tan Y, Zhou J and Li G: Overexpression of Cullin1 is associated with poor prognosis of patients with gastric cancer. Hum Pathol 42: 375-383, 2011

28. Schindl M, Gnant M, Schoppmann SF, Horvat R and Birner P: Overexpression of the human homologue for Caenorhabditis elegans cul-4 gene is associated with poor outcome in node-negative breast cancer. Anticancer Res 27: 949-952, 2007.

29. Wang W, Qiu J, Liu Z, Zeng Y, Fan J, Liu Y and Guo Y: Overexpression of RING box protein-1 (RBX1) associated with poor prognosis of non-muscle-invasive bladder transitional cell carcinoma. J Surg Oncol 107: 758-761, 2013.

30. Yang D, Li L, Liu H, Wu L, Luo Z, Li H, Zheng S, Gao H, Chu Y, Sun Y, et al: Induction of autophagy and senescence by knockdown of ROC1 E3 ubiquitin ligase to suppress the growth of liver cancer cells. Cell Death Differ 20: 235-247, 2013.

31. Jia L, Bickel JS, Wu J, Morgan MA, Li H, Yang J, Yu X, Chan RC and Sun Y: RBX1 (RING box protein 1) E3 ubiquitin ligase is required for genomic integrity by modulating DNA replication licensing proteins. J Biol Chem 286: 3379-3386, 2011.

32. Schlabach MR, Luo J, Solimini NL, Hu G, Xu Q, Li MZ, Zhao Z, Smogorzewska A, Sowa ME, Ang XL, et al: Cancer proliferation gene discovery through functional genomics. Science 319: 620-624, 2008

33. Harper JW, Elledge SJ, Keyomarsi K, Dynlacht B, Tsai LH, Zhang P, Dobrowolski S, Bai C, Connell-Crowley L and Swindell E: Inhibition of cyclin-dependent kinases by p21. Mol Biol Cell 6: 387-400, 1995.

34. Yang X, Pan Y, Qiu Z, Du Z, Zhang Y, Fa P, Gorityala S, Ma S, Li S, Chen C, et al: RNF126 as a biomarker of a poor prognosis in invasive breast cancer and CHEK1 inhibitor efficacy in breast cancer cells. Clin Cancer Res 24: 1629-1643, 2018.

35. Jiao S, Liu W, Wu M, Peng C, Tang H and Xie X: Nrdp1 expression to predict clinical outcome and efficacy of adjuvant anthracyclines-based chemotherapy in breast cancer: A retrospective study. Cancer Biomark 15: 115-123, 2015.

36. Tian YF, Chen TJ, Lin CY, Chen LT, Lin LC, Hsing CH, Lee SW, Sheu MJ, Lee HH, Shiue YL, et al: SKP2 overexpression is associated with a poor prognosis of rectal cancer treated with chemoradiotherapy and represents a therapeutic target with high potential. Tumor Biology 34: 1107-1117, 2013.

37. Gass P, Lux MP, Rauh C, Hein A, Bani MR, Fiessler C, Hartmann A, Häberle L, Pretscher J, Erber R, et al: Prediction of pathological complete response and prognosis in patients with neoadjuvant treatment for triple-negative breast cancer. BMC Cancer 18: 1051, 2018

This work is licensed under a Creative Commons Attribution-NonCommercial-NoDerivatives 4.0 International (CC BY-NC-ND 4.0) License. 